\title{
Conocimiento, percepción y actitud del personal de salud del Hospital Zacamil sobre la vacuna contra la influenza y su aplicación como medida de bioseguridad
}

Dr. Miguel Elas

migelas@yahoo.com.mx

Recepcionado: 20 abril, 2013 / Aceptado: 14 de julio, 2013

\section{RESUMEN}

La vacunación al personal prestador de servicios de salud es de importancia como medida de seguridad laboral debido a: el riesgo que corren de contraer enfermedades infectocontagiosas por la exposición permanente con enfermos tanto directamente en la atención a los mismos o indirectamente en el desarrollo de sus funciones en el ámbito hospitalario; prevención en de diseminación de la enfermedad intrahospitalariamente (evitando brotes epidémicos) y extrahospitalariamente por el riesgo de convertirse en multiplicadores de la infección, al ser portadores y estar contagiados, a pacientes, compañeros de trabajo, familiares y personas en contacto con él; y asegurar la prestación, continua y regular, del servicio de salud, por el riesgo de un ausentismo laboral por enfermedad o cierre de algunas áreas por brotes o epidemias. Sin embargo, la vacunación no es aceptada por muchos de ellos y hasta el momento se desconocen las razones del rechazo de la vacunación, por lo cual se estudió este fenómeno en el personal de salud clave del Hospital Zacamil durante los meses de mayo a agosto del 2011 y a partir de estos hallazgos identificar los puntos clave que ayuden a revertir esta problemática. El Objetivo General del presente estudio es: Determinar el conocimiento, percepción y actitud del personal de salud del Hospital Zacamil sobre la vacuna contra la influenza y su aplicación como medida de bioseguridad.

Palabras claves: vacunación, influenza, personal médico, seguridad laboral, Hospital, Zacamil.

\section{INTRODUCCIÓN}

La inmunización es reconocida como una de las intervenciones preventivas más efectivas desarrolladas y probadas en Salud Pública. Con su uso masivo, su éxito ha sido demostrado en la reducción notoria de la incidencia de importantes enfermedades infecciosas como sarampión, tétanos, difteria, poliomielitis, coqueluche, síndrome de rubéola congénita, enfermedad invasora por Haemophilus influenza tipo b (Hib), e incluso logrando la erradicación de la viruela. Dentro de este marco, la vacunación ha sido una de las estrategias preventivas recomendadas por los organismos mundiales para que sea adoptada por todos los países ya sea para el control, prevención, eliminación o erradicación de las enfermedades inmunoprevenibles. Como parte de estas estrategias de vacunación, en El Salvador, se recomienda la vacunación a grupos de riesgo considerando para ello los factores de vulnerabilidad, riesgo de contagio y/o exposición a la enfermedad, así como los criterios de factibilidad, uso eficiente y racional de los recursos disponibles. De esta manera se clasifica al personal prestador de servicios de salud como un grupo prioritario a ser vacunado por el riesgo que tienen de contraer enfermedades infecciosas inmunoprevenibles debido a su ocupación y convertirse en potenciales transmisores de la enfermedad dentro y fuera de los servicios de salud. Las razones por las cuales el personal de salud debe de ser vacunado responden a los propósitos siguientes:

a. Como medida de seguridad laboral: Por el riesgo de contagio de enfermedades infecciosas que tienen al estar inmersos en un ambiente laboral que implica un contacto permanente directo o indirecto con enfermos, con sus fluidos corporales (orina, sangre, heces, saliva). Así como la manipulación de los desechos bioinfecciosos y uso o manipulación de objetos corto punzante durante la prestación de los servicios de salud y el involucramiento de sus servicios auxiliares.

b. Como medida de protección social: Debido el riesgo de perder la continuidad de funcionamiento regular y normal de los servicios de salud brindados a la población por la disminución de la capacidad instalada de recursos humanos debido a las 
inasistencias por enfermedad, incapacidades o aislamientos necesarios del personal de salud enfermo, el cual puede llegar a ser tan significativo que amerite el cierre parcial o total de un servicio o de un establecimiento de salud en momentos de brotes o epidemias con los consiguientes efectos en la sociedad que quedara desatendida oportunamente ante la demanda.

c. Como medida de responsabilidad social: Debido al riesgo de contraer y convertirse en fuente de transmisión de la infección hacia otros pacientes, familia, amigos o personas en contacto cercano. Por lo que su vacunación implicaría la protección indirecta hacia terceros.

Sin embargo, a pesar de ofrecer los servicios de vacunación al personal de salud en el propio lugar de trabajo, en la práctica esta no es aceptada por la mayoría de estos y hasta el momento se desconocen las razones que hacen que el personal de salud rechace la vacunación. Por tal motivo es de fundamental importancia conocer la percepción que el personal de salud tiene frente a la vacunación y a partir de estos hallazgos identificar los puntos clave, que limitan la vacunación y poder establecer una estrategia de intervención, a través del programa ampliado de inmunizaciones, para revertir esta tendencia y mejorar las coberturas de vacunación, logrando una adecuada seguridad laboral, disminuir el potencial impacto de pérdida de la continuidad de la prestación de los servicios durante períodos de brotes de enfermedad entre trabajadores de salud y proteger a los mismos trabajadores de salud y a sus pacientes, familiares y amigos o personas cercanas de enfermar.

Esta investigación es importante porque devela la percepción, conocimiento y actitud que los trabajadores de salud tienen sobre el riesgo de enfermar y de prevenir la enfermedad, considerando a la vacunación como una de las medidas efectivas de prevención de enfermedades. Además, permite entender la naturaleza de la contradicción entre conocimientos, creencias, itos versus actitud y la práctica en la aceptación o rechazo de la vacunación.
Por tanto se estudiará este fenómeno relacionado principalmente con la vacuna contra la influenza en el Hospital Zacamil durante los meses de mayo a agosto del 2011.

\section{MATERIALES Y MÉTODOS}

Se realizó una investigación cualitativa de tipo transversal, para comprender y analizar la percepción de la vacunación en el personal de salud y responsables de implementar la política de vacunación (tomadores de decisión) dentro de su dinámica laboral y seguridad laboral. La ventaja de este tipo de investigación es que permitió comprender la naturaleza de la actitud de los profesionales de la salud frente a ser vacunados y su significado, comprendiendo además los contextos estructurales y situacionales, identificando la naturaleza profunda de las realidades, su sistema de relaciones, y su estructura dinámica.

Técnicas, procedimientos y fuentes de obtención de la información: Se realizó una entrevista a profundidad para destacar los conceptos y nociones centrales usadas para plantear los supuestos teóricos e hipotéticos en relación a la vacunación y sus prácticas, en esta entrevista se indagó lo siguiente:

- Grado de conocimiento sobre los procesos de control de calidad y seguridad, durante la adquisición de la vacuna y capacitaciones recibidas.

- Percepción sobre las razones por las cuales acepta o no ser vacunado.

- Concepción del personal de salud sobre beneficio -riesgo de la vacunación y enfermar (sus niveles de exposición a enfermedades).

- Accesibilidad de la vacuna a los trabajadores de salud.

- Percepción de la confianza del personal de salud hacia la calidad, seguridad y efectividad de la vacuna y las políticas de vacunación.

- Actitud frente a la vacunación para la prevención de la enfermedad y como medida de bioseguridad.

Para iniciar con la investigación se solicitó la autorización de las autoridades del nivel central y 
las del Hospital Zacamil. Basados en el listado del personal de salud y censos de vacunación se seleccionó a los actores claves por categorías a entrevistar, junto al apoyo de los responsables de ejecución de la medida de vacunación en el hospital. Adicionalmente, se identificó al personal clave tomador de decisión y responsable de implementar la vacunación en el hospital. Se hizo un cronograma de trabajo para entrevistas, previa cita, con el personal seleccionado que cumpliera con los criterios de inclusión y exclusión.

Se ubicó un lugar físico que cumpliera las condiciones de privacidad y tiempo suficiente para realizar las entrevistas las cuales tuvieron una duración promedio de 20 minutos, la cual incluye la parte introductoria. Si una entrevista era interrumpida o no se realizaba en la fecha prevista, esta se volvía a reprogramar las veces necesarias hasta completarla.

\section{RESULTADOS}

\section{Conocimiento y la percepción de los beneficios y riesgos que el personal de salud, tiene sobre la vacuna y sobre el riesgo de enfermar en el ámbito laboral}

\section{Actores que aceptan la vacuna}

El 100\% percibe que la vacunación tiene beneficio y es efectiva, porque "evita, previene y protege contra muchas enfermedades". También consideran que "no tiene muchas reacciones adversas", y que se presentan "reacciones leves a moderadas".

El 71\% percibe que la mayoría del personal de salud acepta la vacunación contra la influenza "para que no les de gripe y porque no está relacionado con neuropatías". Sin embargo un $29 \%$ percibe que "la mayoría del personal de salud no acepta la vacunación", "porque las enfermeras tienen que obligar a la gente".

El $71 \%$ percibe que la campaña de vacunación contra la influenza en el hospital es efectiva porque protege y la mayoría se la aplica, pero el $29 \%$ no la considera efectiva porque "no hay suficiente concientización en el personal y esa es la parte que no se trabaja en la campaña". Lo que es consistente con el $29 \%$ que percibe que "la mayoría del personal de salud no acepta la vacunación" por falta de este convencimiento o concientización.

El $86 \%$ sabe que existen controles de calidad o medidas precautorias que el MINSAL realiza para la compra de las vacunas y como parte de esto "les envían reportes de la calidad de las vacunas recibidas y las que tienen en almacén", pero un $14 \%$ refiere que no conoce.

El $100 \%$ no conoce o no ha tenido experiencias de vacunas de mala calidad adquiridas por el MINSAL y aplicadas a la población. Esto hace alusión a la reafirmación de la confianza en las vacunas por tal razón se acepta su aplicación. Además, consideran que "debido a su trabajo tiene mayor riesgo de contraer influenza por la exposición a pacientes enfermos diariamente".

El 86\% considera que "está en riesgo de convertirse en potencial transmisor de enfermedad a otras personas debido a su ocupación", esto denota el nivel de conocimiento sobre el riesgo $y$ bioseguridad que debe tener para evitar propagar la enfermedad dentro y fuera del hospital, pero un $14 \%$ considera que "no está en riesgo de convertirse en potencial transmisor de enfermedad a otras personas debido a su ocupación", lo que deja entrever la subestimación de la exposición y la falta de percepción de riesgo alguno. Perciben que los medicamentos tienen más riesgo de producir reacciones adversas (86\%) en relación a las vacunas y un $14 \%$ refieren que ambos son iguales en sus riesgos de ocasionar reacciones adversas (medicamentos y vacunas).

\section{Actores claves que rechazan la vacunación}

Un $14 \%$ cree que la vacunación tiene beneficios para la prevención e inmunización contra enfermedades junto a otro tipo de recomendaciones, pero el $86 \%$ no cree que la vacunación tenga beneficio alguno "no sirve para que se van a vacunar", o lo justifican al seguir el ejemplo de otros y decir que "algunos médicos especialistas no se vacunan porque tienen muchas reacciones adversas". El 14\% manifiestan que existe beneficio en la vacunación pero "junto a otro 
tipo de recomendaciones" pero la rechazan como medida preventiva y bioseguridad siendo esto una actitud de ruptura.

El 100\% considera que "la vacunación tiene muchas reacciones adversas, leves, moderadas, gripe, calentura y dolores de hueso y parálisis". Es de hacer notar que la percepción sobre las reacciones leves y moderadas cambia en este grupo, las cuales las consideran como eventos importantes ajenos al proceso normal y esperado de la inmunización y por tanto perjudiciales para la salud y expresan que hay eventos graves poniendo como ejemplo la parálisis.

Un $71 \%$ percibe que la mayoría del personal de salud no acepta la vacunación contra la influenza esto debido a "los eventos adversos y a que hay enfermeras que no se dejan vacunar y tienen años de trabajar en eso".

El $29 \%$ percibe que la mayoría del personal de salud acepta la vacunación contra la influenza "por el riesgo de contacto con enfermos", esto hace alusión a una actitud de conciencia y conocimiento sobre el beneficio de la vacunación y seguridad laboral existente en parte del personal de salud, pero también deja en claro una actitud de denegación, porque no mencionan como uno de los condicionantes para la aceptación de la vacuna las medidas administrativas adoptadas donde tuvieron que firmar como constancia de no haber aceptado la vacunación, evadiendo de esta forma evitar mayores problemas sobre comentarios negativos al comportamiento tomado previamente de rechazo de la vacunación.

El $71 \%$ percibe que la campaña de vacunación contra la influenza en el hospital no ha sido efectiva porque "no todo el personal lo acepta", pero el $29 \%$ si percibe que la campaña de vacunación contra la influenza en el hospital ha sido efectiva "porque bastante gente se deja". Además, nadie sabe $(100 \%)$ si existen controles de calidad o medidas precautorias que el MINSAL realiza para la compra de las vacunas.
El $86 \%$ considera que debido a su trabajo no tiene mayor riesgo de contraer influenza y no perciben a la vacunación como parte de una medida de seguridad laboral. Esto coincide con la actitud de rechazo de la vacunación lo que hace alusión a un desconocimiento sobre exposición a riesgos laborales y salud ocupacional. El 14\% considera que si tiene mayor riesgo de contraer influenza debido a que esta con un "trato directo a la gente y porque trabaja en un hospital"; esto hace alusión a un grado de conciencia sobre el riesgo laboral en el que están inmersos al estar en contacto permanente con un agente.

El $86 \%$ considera que el producto que tiene más riesgo de reacciones adversas son los medicamentos y un $14 \%$ ambas (medicamentos y vacunas). Sin embargo al preguntar que producto considera da mayor beneficio para la salud, el $86 \%$ considera que los medicamentos y un $14 \%$ considera que ambas, esto hace alusión a una contradicción ya que a pesar de que los medicamentos tienen mayores reacciones adversas prefieren someterse a estos antes de someterse a la vacunación.

Con respecto a la vacuna contra la influenza estacional, ¿qué considera que tiene mayor peso los beneficios en la prevención o las reacciones adversas? El $86 \%$ considera que las reacciones adversas tienen mayor importancia y son más significativas que el poco beneficio en prevención por la vacunación, porque son muchas aunque leves; un $14 \%$ considera que los beneficios tienen mayor peso y son mayores que las reacciones adversas.

Nivel de confianza que el personal de salud, atribuye a la vacuna y a la política de vacunación como medida de bioseguridad

\section{Actores claves que aceptan la vacunación}

El 57\% considera que "no es conveniente crear una ley que obligue a vacunarse" y que "debe ser voluntario". 
Esto se puede interpretar como una actitud de denegación, evitando entrar en confrontaciones con los otros actores al establecer un estándar de política de vacunación independiente de la voluntad hacia la aceptación o rechazo; pero el $43 \%$ considera que "está bien" establecer dicha política para aplicársela a todos por obligación y no voluntariamente.

El $86 \%$ refiere tener confianza en la política de vacunar contra la influenza a todo el personal que labora en el hospital, lo que se relaciona con el $43 \%$ que menciona que "está bien" establecer la política de vacunar a todos, esto apoya también la actitud de denegación de los actores que opinaron que la vacunación "debe ser voluntario". Un 14\% refirió no tener confianza en esta política, lo que denota que dentro de los actores claves un porcentaje menor no están completamente convencidos sobre la vacuna por lo que existieron otros factores que condicionaron su aceptación.

El $100 \%$ considera que la calidad de la vacuna adquirida por el Ministerio de Salud es buena porque "no ha habido reacciones adversas y porque la gente no se enferma". En este grupo deja claro que las reacciones leves no son consideradas como eventos adversos dañinos o perjudiciales que atenten contra la salud de quien se la aplica, por tanto todos consideran que la calidad de las vacunas es buena. El $86 \%$ cree que hay buen control de calidad por parte del Ministerio de Salud en las vacunas que compra "porque tiene laboratorio de control de calidad para ver que todo medicamento este en buen estado". El 14\% cree que no porque "desconoce y no puede saberlo". Esto es una actitud de ruptura en el cual acepta desconocer los procesos de control de calidad de las vacunas y se relaciona con el $14 \%$ que no tiene confianza en la política de vacunación.

El $86 \%$ considera que las vacunas donadas al MINSAL son de buena calidad, porque "tienen buen control de calidad y tienen más calidad que las que el gobierno compra", esto denota un falta de conocimiento en los procesos de adquisición ya que tanto la vacuna donada como comprada provienen de los mismos laboratorios productores. Un $14 \%$ no sabe la calidad de las vacunas donadas.

\section{Actores claves que rechazan la vacunación}

El $100 \%$ no está de acuerdo con la política de establecer que todo quien labora en un hospital sea vacunado contra la influenza y menciona que esto "debe ser voluntario por los efectos secundarios". Además, no tiene confianza en la política de vacunar contra la influenza a todos en el hospital debido a los "eventos adversos".

El $71 \%$ considera que la calidad de la vacuna adquirida por el Ministerio de Salud es de mala y baja calidad y un $29 \%$ la considera de buena calidad. El $86 \%$ cree que no hay buen control de calidad de las vacunas por parte del MINSAL en las vacunas que se compran, debido a que "compran lo más barato", por lo que atribuye la calidad de la vacuna al costo de la misma (la creencia de asociar el valor del producto con la calidad del mismo); sin embargo el $14 \%$ cree que si hay un "buen control de calidad" debido a que "por experiencia nunca ha habido problema". Esto denota cierta confianza basado en la experiencia que tiene sobre las vacunas.

El $71 \%$ dice que la calidad de las vacunas donadas al MINSAL "no son buenas porque las donan, porque no las aceptan en otras partes y están a punto de vencerse". El 29\% considera que la calidad de las vacunas donadas al MINSAL "es buena", esto hace alusión a que las causas de rechazo de vacuna son otras y no la confiabilidad de las mismas o denota una actitud de denegación por temor a decir lo contrario o cosas de las cuales no tiene pruebas.

El $86 \%$ considera que la vacuna contra la influenza no es efectiva ni segura, porque "hay más enfermos y las personas siempre se enferman", y el $14 \%$ no sabe. El $71 \%$ cree que las vacunas no son lo suficientemente investigadas antes de salir al mercado y que "las mandan a estos países para probarlas". Un $29 \%$ dice que no sabe.

\section{DISCUSIÓN}

Los actores claves, que aceptan la vacunación, los médicos y los que implementan la política de vacunación, así como la mitad de los actores claves no médicos, reconocen que la vacunación tiene 
beneficio para la prevención de enfermedades. Esto denota que existe un grado de conocimiento general sobre las vacunas, sin embargo este conocimiento general sobre prevención por las vacunas no es tomado como un punto de vital importancia en términos de evidencia de protección a la salud, al relacionarla con la vacuna contra la influenza, ya que mencionan que no se la aplican porque "no la considero necesaria". Y, aunque consideran que están en riesgo de contraer la influenza debido a su ocupación, hay una contradicción, no identifican a la vacunación como un beneficio en el impacto de la prevención, demostrando esta desconfianza en la eficacia de la vacuna al referirse a que a pesar de la vacunación "hay más enfermos" y "las personas siempre se enferman".

Además, existe un reforzamiento de esta creencia de desconfianza de la efectividad de la vacunación en el modelaje del personal de salud el cual se cree más capacitado y con mayor experiencia en el área de vacunación y por tanto con mayor confianza de creerle, lo que se demuestra cuando se refieren a que "hay enfermeras que no se dejan vacunar y tienen años de trabajar en eso". Por tal motivo esto refleja la prueba fehaciente de los conocimientos y la confianza atribuida a este grupo y que determina una actitud a seguir, de rechazo de la vacunación por un grupo importante de la población hospitalaria.

Hay que recordar que "La confianza es la expectativa que surge dentro de una comunidad de comportamiento normal, honesto y cooperativo, basada en normas comunes, compartidas por todos los miembros de dicha comunidad". Las sociedades parecen estar organizadas por medio de contratos voluntarios emocionales entre los diferentes grupos intervinientes. No obstante, el hecho de que permanezcan unidos no se puede explicar sino es a través de la figura de la confianza.

En este sentido, las decisiones con respecto a los riesgos son tomadas siguiendo el esquema fijado por las normas y la moral; y no necesariamente de un análisis racional causa-efecto. (Korstanje, 2009) Los que rechazan la vacunación tienen la creencia de que la vacuna no tiene beneficios ya que, "hay más enfermos" y "las personas siempre se enferman" por lo que no es efectivo. Esto está probado porque "hay enfermeras que no se dejan vacunar y tienen años de trabajar en eso"; por tanto aunque se tenga la percepción de riesgo de enfermarse debido a su trabajo, la vacunación no se considera como una medida aceptable, e incluso algunos de ellos llegan a mencionar que la vacuna "no sirve para nada". Esto está en un franca contradicción con la opinión de los actores claves responsables de implementar la política de vacunación dentro del hospital quienes consideran que la vacuna contra la influenza es eficaz y segura y su argumento lo amparan en que "está probado por estudios científicos", lo que denota una marcada brecha de conocimiento entre los que implementa la política y el resto del personal hospitalario.

Existe una marcada diferencia sobre la percepción de la seguridad de la vacuna contra la influenza entre el grupo que acepta la vacunación y el grupo que rechaza la vacunación. El grupo que acepta la vacunación, percibe que la vacuna es segura y no percibe como reacciones adversas, las reacciones leves y moderadas que se pueden presentar después de la vacunación ("calentura, dolores de hueso, gripe") y que son descritos por el fabricante de la vacuna. Más bien son concebidas como situaciones regulares, normales y esperadas del proceso de vacunación por tanto no significan ningún riesgo para la salud, no representan ninguna peligrosidad y que su aparecimiento es despreciable frente al beneficio obtenido por la vacunación razón por la cual consideran que la vacunación contra la influenza no tiene eventos adversos.

Por el contrario, el grupo que rechaza la vacunación tiene una percepción negativa magnificada $y$ exagerada de los eventos leves y moderados considerándolos de mucha importancia para la salud. Por tanto con un alto grado de peligrosidad hasta el punto de llegar a ser catastróficos para la población, por tanto un problema de seguridad de la vacuna misma, por lo que no tiene confianza en 
lo descrito por el fabricante de la vacuna como reacciones esperadas y normales.

Esto se ve fundamentado en el hecho de que considera que la vacuna "no es investigada lo suficiente antes de salir al mercado" y "las mandan a estos países para probarlas", refiriéndose así a un ensayo de la vacuna con la población de los países en vías de desarrollo como El Salvador. Esta falta de confianza en la calidad y la seguridad de la vacuna, los actores que rechazan la vacunación, la reafirman al considerar de "mala o baja calidad la vacuna comprada por el MINSAL" porque "no hay buen control de calidad por parte del MINSAL" y "compran lo más barato". Sin embargo refieren que no han tenido experiencia ni conocen de algún caso de evento adverso grave sucedido debido a la vacuna en el país, ni tampoco conocen información científica ni han sido capacitados sobre vacunas.

Un porcentaje de los que aceptan la vacuna y la mayoría de los que rechazan la vacunación coinciden en que la campaña no es efectiva pero la causa de esto es distinta. Los que aceptan la vacunación dicen que es por "falta de concientización y capacitación...", mientras que los que rechazan dicen que es porque "la vacuna no es efectiva y no sirve para nada y que el personal de salud no acepta la vacunación si no que se ven obligados a vacunarse". La obligación a vacunarse parece ser un punto común de convergencia entre estos grupos por lo que la aceptación de la vacunación ha sido un proceso condicionado por la concientización y percepción del riesgo administrativo y no a la concientización y percepción del riesgo de la enfermedad, la bioseguridad y calidad de la vacuna.

La percepción puede hacer referencia a un conocimiento, a una idea o a la sensación interior que resulta de una impresión material hecha en nuestros sentidos. La percepción del riesgo está íntimamente relacionado con la percepción de confianza y temores, y con la forma en que se estructura y articulan estas variables, siendo estas imágenes lo que mueven y condicionan a las personas a actuar de una y otra forma, independientemente que estas sean reales 0 aparentes.
Aunque existe una percepción de riesgo laboral no existe confianza en la política de vacunación, ni sobre la seguridad y calidad de la vacuna lo que denota un desconocimiento de la vacuna, ya que al mismo tiempo no encuentran evidencia de la inseguridad o peligrosidad de la vacuna más allá de los comentarios negativos escuchados.

No hay un conocimiento completo y claro sobre los procesos de compra y control de calidad para la adquisición de la vacuna en los grupos de actores que rechazan, lo que se evidencia al responder "no sé", "no tengo como saberlo", "desconozco" y "no", así como de los requisitos y pruebas realizadas a la vacuna y evaluadas y monitoreadas por la OMS y OPS, siendo que estas entidades son los mecanismos a través de los cuales se adquiere la vacuna para ser usada en el país.

Así también los conocimientos sobre la evaluación post mercadeo y su vigilancia de garantía de calidad, lo que hace tambalear los "conocimientos científicos" de los actores frente a la influencia magnificada y exagerada de la promoción negativa difundidos por rumores: "dolor de hueso, calentura, gripe, siempre se enferma, no sirve para nada, inflamación de brazo, dolor de brazo, da parálisis", esto se ve reforzado ante la evidencia de un pobre conocimiento sobre las vacunas y falta de búsqueda de información científica más allá de los medios tradicionales y comunes de los distintos medios de comunicación.

Esto denota la falta de estudio sobre los temas de vacunas; esto se reafirma al mencionar que en el último año no han recibido capacitación sobre vacunas y lo que proponen para mejorar la confianza en la vacunación es: "dar charlas orientadas a aumentar la confianza de la vacuna incluyendo la discusión de los mitos y creencias, calidad y seguridad de la vacuna y los procesos de adquisición e investigaciones, yunificando a todos los departamentos del hospital".

\section{CONCLUSIONES}

Existe una diferencia importante en la percepción y conocimiento entre el grupo que acepta y el que rechaza, en cuanto a:

a) Las reacciones adversas: varían desde una situación normal y esperada hasta una situación 
con dimensiones catastróficas, la cual se relaciona con las creencias y mitos, exagerando o tergiversar su significado e importancia en términos de efectividad, seguridad y calidad.

b) El nivel de peligrosidad en la exposición: considerando que la exposición laboral a la enfermedad es más benigna que la exposición a los daños catastróficos de la vacunación, el cual es potencializado por el pensamiento médico curativo.

c) El riesgo laboral de enfermar: que va desde la creencia y confianza de no estar expuestos a riesgo, dominada por un desconocimiento de dichos procesos, hasta la percepción de estar conscientes de su propio riesgo laboral.

Hay un alto grado de desconocimiento de los procesos de control de calidad y adquisición de la vacuna, además de desconocer estudios realizados sobre la seguridad de la vacuna y efectos adversos, política de vacunación, bioseguridad y una desinformación debido a la influencia de rumores divulgados por distintos medios de comunicación.

Existe una brecha de conocimiento entre los actores que implementan la política de vacunación y los demás actores, por lo tanto una percepción y actitud distinta a la aceptación de la vacunación con mayores fundamentos que el resto de actores.

Los rumores, los mitos, creencias y las ideas comúnmente falsas, sostenidas sobre la vacuna contra la influenza, son variadas y tienen poca diferencia entre los actores médicos y no médicos.

El desconocimiento, los rumores, los mitos, creencias y las ideas comúnmente falsas, sostenidas sobre la vacuna contra la influenza esta significativamente asociada con el alto grado de duda en la calidad de la vacuna. Por tanto de poca seguridad y poca efectividad, entrelazado con la desconfianza en la política de vacunación que manifiestan los actores que rechazan la vacunación.

El déficit en el conocimiento general de la vacunación y sus beneficios, actúa como un factor condicionador de la percepción y la actitud a la vacunación lo que obstaculiza significativamente el aumento de las tasas de cobertura de vacunación.

La aceptación de la vacunación en un porcentaje mínimo en el grupo que la rechaza ha sido un proceso condicionado por un riesgo de acción administrativa y no a la concientización y percepción del riesgo de la enfermedad, la bioseguridad y calidad de la vacuna.

\section{BIBLIOGRAFÍA}

Abarca V, K. (2007). Vigilancia de eventos adversos a vacunas. Revisata Chilena de Infectología, 24 (1), 53-56.

Abramson, Z. H., \& Levi, O. (2008). Influenza vaccination among primary health care workers. ScienceDirect(Vaccine 26), 2482 - 2489.

AlvarezGarcía, F. (2000).Medicina TV.com profesional. Recuperado el 15 de marzo de 2009, de curso de vacunas: http://profesional.medicinatv.com/fmc/cursova cunas/vacuna9/parte33.asp

ArgimonPallás, J. M. (2004). Métodos de investigación clínica y epidemiológica (Tercera ed.). Madrid, Madrid, España: ELSEVIER.

Asociación Española de Vacunología. (11 de Junio de 2010). Vacunas. org. Recuperado el 21 de abril de 2011, de Vacunas.org: http://www.vacunas.org/es/infoprofesionales/n oticias/114363

Carmen WF, E. A. (2000). Effects of influenza vaccination of health care workers on mortality of elderly people in long-term care: a ramdomized controlled study. Lancet (395 (9198)), 7 - 93.

CDC. (2006).Influenza vaccination of health-care personnel. Recommendations of the healthcare infection control pracices advisory committee (HICPAC) and the advisory committee on inmunization practices (ACIP). MMWR, 55(RR02):1-16. 
Díez-Delgado Rubio, M. L. (1996). Percepción de la vacunación por parte de los padres. Anales españoles de pediatría Hospital "Torrecárdenas" , 45 (2), 129 - 132.

Frank E, R. R. (2000). Correlates of physicians' prevention-related practices. Findings from the women physicians' health study. Arch FamMed, 9:359-67.

Fundación Española para la Ciencia y Tecnología (FECYT). (2010). Fundación Española para la Ciencia y Tecnología (FECYT). Recuperado el 22 de abril de 2011, de Fundación Española para la Ciencia y Tecnología (FECYT): http://www.upf.edu/pcstacademy/ docs/Apunt esFecyt.pdf

Hayward AC, H. R. (2006). Effectiveness of an influenza vaccine programme for care home staff to prevent death, morbidity, and health service use among residents: cluster randomized controlled trial. British Medical Journal, do1:10.1136/bmj.39010.581354.55., 333:1241.

Heimberger T, C. H. (1995). Knowledge and attitudes of healthcare workers about influenza: why are they not getting vaccinated? Infection Control Hospital Epidemiology, 16, 412 - 414. J.

Juio, P. (1998). Introducción a la metodología de la investiación científica (Tercera ed.). (C. d. Salud, Ed.) Managua, Nicaragua: Litografía y tipografía rojas.

Korstanje, M. (21 de enero de 2009). Universidad Complutense. Recuperado el 22 de abrilde 2011, de Universidad Complutense: http://www.ucm.es/info/nomadas/21/maxkorst anje.pdf 